\title{
Gauge invariance of the vector meson mass in the Coleman-Weinberg model
}

\author{
Haojie Shen $\odot,{ }^{1, *}$ Yu Cheng $\odot,{ }^{1,2, \dagger}$ and Wei Liao $\odot^{1, *}$ \\ ${ }^{1}$ Institute of Modern Physics, School of Sciences, East China University of Science and Technology, \\ 130 Meilong Road, Shanghai 200237, People's Republic of China \\ ${ }^{2}$ Tsung-Dao Lee Institute, and School of Physics and Astronomy, Shanghai Jiao Tong University, \\ Shanghai 200240, People's Republic of China
}

(Received 24 January 2021; accepted 29 March 2021; published 22 April 2021)

\begin{abstract}
We revisit the problem of the gauge invariance in the Coleman-Weinberg model in which a $U(1)$ gauge symmetry is driven spontaneously broken by radiative corrections. It was noticed in previous work that masses in this model are not gauge invariant at one-loop order. In our analysis, we use the dressed propagators of scalars which include a resummation of the one-loop self-energy correction to the tree-level propagator. We calculate the one-loop self-energy correction to the vector meson using these dressed propagators. We find that the pole mass of the vector meson calculated using the dressed propagator is gauge invariant at the vacuum determined using the effective potential calculated with a resummation of daisy diagrams.
\end{abstract}

DOI: 10.1103/PhysRevD.103.076016

\section{INTRODUCTION}

One of the subtle problems in quantum field theory (QFT) is the gauge invariance of physical quantities. It is generally believed that physical quantities, such as the $S$-matrix elements, the physical masses, the energy density of a physical state, and the decay rate of a false vacuum, are gauge invariant although the quantum field theory can be quantized in an arbitrary gauge. One quantity often encountered in QFT is the effective potential which is usually interpreted as the energy density just as the potential is usually interpreted as the energy density.

However, it was found that the effective potential in the Coleman-Weinberg (CW) model [1], which is a model of massless scalar quantum electrodynamics (QED) with symmetry broken by radiative corrections, is not gauge invariant [2]. This has raised concerns about how the gauge invariance is achieved in physical quantities in scalar QED and in general scalar gauge theories and what is the meaning of the effective potential. Many attempts have been made to clarify this problem in different models for various quantities [3-22], e.g., for the decay rate of the false vacuum $[14,15,18,19]$.

\footnotetext{
*haojieshen@mail.ecust.edu.cn †chengyu@mail.ecust.edu.cn

liaow@ecust.edu.cn
}

Published by the American Physical Society under the terms of the Creative Commons Attribution 4.0 International license. Further distribution of this work must maintain attribution to the author(s) and the published article's title, journal citation, and DOI. Funded by SCOAP .
One interesting observation is that although the effective potential is in general not gauge invariant, but the minimum value of the effective potential turns out to be gauge invariant [3]. So the minimum value of the effective potential is indeed a physical quantity. Another interesting observation is that the pole masses in the $\mathrm{CW}$ model are not gauge invariant at one-loop order, but the ratio of the pole masses of the scalar meson and the vector meson are gauge invariant [4]. These results are encouraging but are still far from a clear clarification. In particular, the gauge dependence of the pole masses in the CW model is apparently not satisfactory. It is a puzzling result. The gauge independence of the minimum of effective potential is also not easy to see clearly although the argument given in [3] looks straightforward.

It was noticed in $[3,5]$ that an infinite series of diagrams, the so-called daisy diagrams, can give contributions of the same order of the gauge-dependent part in the one-loop contribution to the effective potential and these diagrams should be taken into account in a consistent analysis [6]. In was shown that these contributions can also be evaluated by summing the daisy diagrams into the dressed propagators of scalar fields [7]. A recent publication [9] shows that after a careful calculation of the daisy diagrams the gauge invariance of the minimum of the effective potential can be achieved. Actually, daisy resummation also plays an important role in solving other problems, e.g., the gauge dependence problem in finite-temperature field theory [1113] and the infrared problem caused by the massless Goldstone boson [23,24]. In view of these recent advancements, it is natural to expect that this idea can help to solve other remaining problems, e.g., the gauge dependence of the physical mass in the $\mathrm{CW}$ model. 
In this article, we reanalyze the radiative correction to the pole mass of the vector meson in the CW model at one-loop order. We show that the pole mass of the vector meson in the CW model is gauge invariant at one-loop order after including the daisy resummation effects in the propagator and in the effective potential. This result is the novel contribution of the present article. This was argued in [9] to happen, but a concrete calculation has not been done yet. Since this problem has been a puzzle for a long time, it is worth making a detailed presentation although the content may look pedagogical.

The article is organized as follows. In the next section, we quickly review the $\mathrm{CW}$ model and the effective potential after including the resummation effect. We obtain an expression of vacuum using this effective potential. Then we address the resummation effects in the propagators and calculate the one-loop self-energy of the vector meson when including these effects. We find that the gauge dependence in the pole mass of the vector meson is completely canceled at one-loop order after including all these resummation effects. We give some details of our calculation in Appendixes A, B, and C.

\section{THE EFFECTIVE POTENTIAL AND THE VACUUM}

The CW model is a model with a massless scalar field coupled with a $U(1)$ gauge field. The Lagrangian of the $\mathrm{CW}$ model can be written as follows:

$$
\begin{aligned}
\mathcal{L}= & -\frac{1}{4} F_{\mu \nu}^{2}+\frac{1}{2}\left(\partial_{\mu} \phi_{1}-e A_{\mu} \phi_{2}\right)^{2} \\
& +\frac{1}{2}\left(\partial_{\mu} \phi_{2}+e A_{\mu} \phi_{1}\right)^{2}-V(\phi)+\mathcal{L}_{\mathrm{GF}},
\end{aligned}
$$

where $\phi_{1}$ and $\phi_{2}$ are two components of the complex scalar field $\phi, A_{\mu}$ is the $U(1)$ gauge field and $F_{\mu \nu}$ the field strength, and the potential $V$ is

$$
V(\phi)=\frac{\lambda}{24} \phi^{4}
$$

with $\phi^{2} \equiv \phi_{1}^{2}+\phi_{2}^{2} \cdot \mathcal{L}_{\mathrm{GF}}=-\frac{1}{2 \xi}\left(\partial_{\mu} A^{\mu}\right)^{2}$ is the gauge-fixing term. The tree level potential of the $\mathrm{CW}$ model has a minimum at $\phi=0$, where the original symmetry is not broken. However, quantum correction can give rise to an effective potential which can drive the minimum away from the origin, as pointed out in [1]. This leads to symmetry breaking driven by radiative corrections.

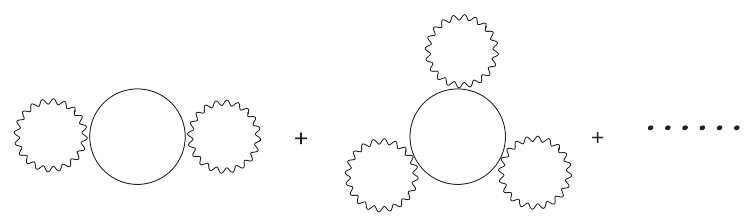

FIG. 1. High-loop graphs that contribute terms proportional to $\frac{e^{10}}{\lambda}$ to the effective potential.

The effective potential can be obtained using the background field method [2]. The effective potential including the leading quantum correction is obtained as

$$
V_{e^{4}}(\hat{\phi})=\frac{\lambda}{24} \hat{\phi}^{4}+\frac{\hbar e^{4}}{16 \pi^{2}} \hat{\phi}^{4}\left(-\frac{5}{8}+\frac{3}{2} \ln \frac{e \hat{\phi}}{\mu}\right),
$$

where $\hat{\phi}$ is a background field taken as the value of $\phi_{1}$, i.e., $\phi_{1}=\hat{\phi}$ and $\phi_{2}=0$, and $\mu$ is the renormalization scale. The effective potential in Eq. (2.3) has been renormalized in the modified minimal subtraction $(\overline{M S})$ scheme. We can see in Eq. (2.3) that if $\lambda \sim \frac{\hbar}{16 \pi^{2}} e^{4}$, the potential can have a minimum for $\phi \neq 0$, and then the symmetry is broken spontaneously driven by quantum corrections.

The effective potential in Eq. (2.3) is gauge invariant. However, there are other terms in the one-loop quantum correction. In particular, there is a term of order $\lambda e^{2}$ which depends on the gauge-fixing parameter $\xi$, as shown in [2]. Since $\lambda \sim e^{4}$ in this model, this gauge dependent term is of higher order, i.e., of order $e^{6}$. As a consequence, the masses obtained using this effective potential are not gauge invariant. It was shown in [4] that the scalar-to-vector mass ratio is gauge independent if including two-loop corrections in the effective potential, but the masses of the scalar meson and the vector meson are still gauge dependent.

It was suggested in $[3,5]$ that all the higher-loop contributions given in Fig. 1, the so-called daisy diagrams, should be included when calculating the effective potential. This is because these diagrams would give a contribution of order $e^{10} / \lambda$ which is of order $e^{6}$ for $\lambda \sim e^{4}$. A resummation of the contributions of these diagrams have been done in [9] and an effective potential is obtained as follows:

$$
V(\hat{\phi})=V_{e^{4}}(\hat{\phi})+V_{e^{6}}(\hat{\phi}),
$$

where $V_{e^{4}}$ is given in Eq. (2.3) and $V_{e^{6}}$ in the $\overline{M S}$ scheme is [9]

$$
\begin{aligned}
V_{e^{6}}(\hat{\phi})= & \frac{\hbar e^{2} \lambda}{16 \pi^{2}} \hat{\phi}^{4}\left(\frac{\xi}{8}-\frac{\xi}{24} \ln \frac{e^{2} \lambda \xi \hat{\phi}^{4}}{6 \mu^{4}}\right) \\
& +\frac{\hbar^{2} e^{6}}{\left(16 \pi^{2}\right)^{2}} \hat{\phi}^{4}\left[(10-6 \xi) \ln ^{2} \frac{e \phi}{\mu}+\left(-\frac{62}{3}+4 \xi-\frac{3}{2} \xi \ln \frac{\lambda \xi}{6 e^{2}}\right) \ln \frac{e \hat{\phi}}{\mu}+\xi\left(-\frac{1}{2}+\frac{1}{4} \ln \frac{\lambda \xi}{6 e^{2}}\right)+\frac{71}{6}\right] \\
& +\frac{\hbar e^{2} \lambda}{16 \pi^{2}} \hat{\phi}^{4}\left(-\frac{\xi}{24}\right)\left[\frac{\hat{\lambda}(\hat{\phi})}{\lambda}+\left(1-\frac{\hat{\lambda}(\hat{\phi})}{\lambda}\right) \ln \left(1-\frac{\hat{\lambda}(\hat{\phi})}{\lambda}\right)\right]
\end{aligned}
$$


where

$$
\hat{\lambda}(\hat{\phi}) \equiv \frac{\hbar e^{4}}{16 \pi^{2}}\left(6-36 \ln \frac{e \hat{\phi}}{\mu}\right) .
$$

The second part in Eq. (2.5) comes from two-loop contributions, and the third part in Eq. (2.5) is the contribution of the resummation of daisy diagrams in Fig. 1. Using this effective potential, it was shown explicitly that the effective potential at its minimum is gauge invariant to order $e^{6}$ [9].

The vacuum expectation value, $\hat{\phi}=v$, can be obtained from extremum condition

$$
\left.\frac{\partial V(\hat{\phi})}{\partial \hat{\phi}}\right|_{\hat{\phi}=v}=0 .
$$

Since $V(\hat{\phi})$ is not gauge invariant, $v$ is not gauge invariant either. However, it can be found that $v$ is gauge invariant at the leading order and is gauge dependent at order $e^{2}$. So we can express $v$ as follows:

$$
v=v_{0}+v_{1}+\cdots,
$$

where $v_{0}$ is the value of $v$ at leading order and $v_{1}$ is the correction at order $e^{2}$. Other higher-order corrections have been neglected. $v_{0}$ can be obtained by considering the extremum condition of $V_{e^{4}}$, i.e.,

$$
\left.\frac{\partial V_{e^{4}}(\hat{\phi})}{\partial \hat{\phi}}\right|_{\hat{\phi}=v_{0}}=0 .
$$

It gives

$$
\lambda-\hat{\lambda}\left(v_{0}\right)=0,
$$

where $\hat{\lambda}$ is given in Eq. (2.6). $v_{0}$ determined using Eq. (2.10) is gauge invariant because the expression of $\hat{\lambda}$ in Eq. (2.6) does not depend on the gauge-fixing parameter $\xi$.

The coupling constants $\lambda$ and $e$ in Eq. (2.10)) should be understood as renormalized at the scale $\mu$. Using the $\beta$ functions of $\lambda$ and $e$ at the leading order [9]

$$
\mu \frac{d}{d \mu} \lambda=\frac{9 \hbar e^{4}}{4 \pi^{2}}, \quad \mu \frac{d}{d \mu} e=\frac{\hbar e^{3}}{48 \pi^{2}},
$$

it is straightforward to show that $v_{0}$ determined in Eq. (2.10)) does not depend on the renormalized scale $\mu$ at the leading order, i.e., at order $e^{4}$. So it is convenient to choose a fixed energy scale $\mu_{X}$ for which the running coupling constants satisfy the following relation [9]:

$$
\lambda\left(\mu_{X}\right)=\frac{\hbar}{16 \pi^{2}} e^{4}\left(\mu_{X}\right)\left\{6-36 \ln \left[e\left(\mu_{X}\right)\right]\right\} .
$$

We can choose $\mu=\mu_{X}$ in Eq. (2.10). Then it is easy to see that

$$
v_{0}=\mu_{X} .
$$

Since $v_{0}$ does not depend on $\mu, v_{0}$ found in this way is the same for an arbitrary value of $\mu$.

Now we can obtain an expression of $v_{1}$. Notice that Eq. (2.9) means

$\left.\frac{\partial V_{e^{4}}(\hat{\phi})}{\partial \hat{\phi}}\right|_{\hat{\phi}=v_{0}+v_{1}}=\left.\frac{\partial^{2} V_{e^{4}}(\hat{\phi})}{\partial \hat{\phi}^{2}}\right|_{\hat{\phi}=v_{0}} v_{1}+O\left(v_{1}^{2}\right)+\cdots$.

Keeping the term linear in $v_{1}$ in Eq. (2.14), we can write Eq. (2.7) as

$$
\left.\frac{\partial^{2} V_{e^{4}}(\hat{\phi})}{\partial \hat{\phi}^{2}}\right|_{\hat{\phi}=v_{0}} v_{1}+\left.\frac{\partial V_{e^{6}}(\hat{\phi})}{\partial \hat{\phi}}\right|_{\hat{\phi}=v_{0}+v_{1}}=0 .
$$

Plugging Eq. (2.5) into Eq. (2.15) and keeping terms of the leading order, we obtain

$$
\begin{aligned}
v_{1}= & \frac{\hbar e^{2}}{16 \pi^{2}} \frac{\mu_{X}}{2}\left[\left(-\xi-\frac{80}{9}\right)-\frac{40}{3} \ln ^{2} e+\frac{94}{9} \ln e^{2}\right. \\
& \left.+\frac{1}{2} \xi\left(\ln \frac{\lambda \xi e^{2}}{6}+\ln \left(1-\frac{\hat{\lambda}(v)}{\lambda}\right)\right)\right] .
\end{aligned}
$$

In Eq. (2.16), the $\ln \left(1-\frac{\hat{\lambda}(v)}{\lambda}\right)$ have been kept as a function of $v=\mu_{X}+v_{1}$ rather than of $\mu_{X}$, because this logarithm diverges at $\hat{\phi}=\mu_{X}$. So Eq. (2.16) can be understood as an iterative expression of $v_{1}$. We can see in Eq. (2.16) that $v_{1}$ is indeed of order $e^{2}$. This expression of $v_{1}$ will be used later when studying the gauge invariance of the vector meson mass.

\section{THE DRESSED PROPAGATOR}

Using the background field $\hat{\phi}$, the effective propagators of scalars at tree level are [4]

$$
\begin{aligned}
& 1 \longrightarrow D_{11}^{0}(k)=\frac{i}{k^{2}-\frac{\lambda}{2} \hat{\phi}^{2}}, \\
& 2 \longleftarrow D_{22}^{0}(k)=\frac{i\left(k^{2}-e^{2} \xi \hat{\phi}^{2}\right)}{k^{4}-\frac{\lambda}{6} \hat{\phi}^{2}\left(k^{2}-\xi e^{2} \hat{\phi}^{2}\right)} .
\end{aligned}
$$




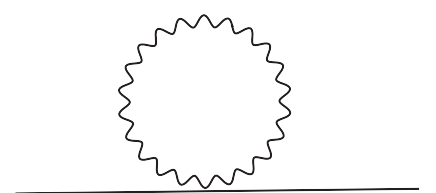

FIG. 2. Transverse vector tadpole graph $A_{\text {loop }}$.

The key point of daisy summation is that the self-energy diagram shown in Fig. 2 is of the same order of tree-level contribution. In fact, a straightforward calculation of this diagram using the dimensional regulation gives

$$
\begin{aligned}
A_{\text {loop }} & =\frac{\hbar}{2} \int \frac{d^{D} k}{(2 \pi)^{D}} 2 i e^{2} g_{\mu \nu} \frac{-i}{k^{2}-e^{2} \hat{\phi}^{2}}\left(g^{\mu \nu}-\frac{k^{\mu} k^{\nu}}{k^{2}}\right) \\
& =\frac{i \hbar}{16 \pi^{2}} e^{4} \hat{\phi}^{2}\left(1-6 \ln \frac{e \hat{\phi}}{\mu}\right),
\end{aligned}
$$

where we have performed subtraction in the $\overline{M S}$ scheme and have eliminated the term proportional to $\Delta_{\varepsilon}=\frac{1}{\varepsilon}-\gamma_{E}+\ln 4 \pi$. One can see clearly in Eqs. (3.1) and (3.2) that the self-energy diagram in Fig. 2 is of the same order of the tree-level mass, which is $\sim \lambda \hat{\phi}^{2} \sim e^{4} \hat{\phi}^{2}$.

This suggests that we should include a geometric summation shown in Fig. 3 in the calculation of quantum corrections $[7,8]$. This summation gives modified propagators, called dressed propagators. For example, for $\phi_{2}$ the summation gives a dressed propagator as follows:

$$
\begin{aligned}
D_{22} & =D_{22}^{0}+D_{22}^{0} A_{\text {loop }} D_{22}^{0}+D_{22}^{0} A_{\text {loop }} D_{22}^{0} A_{\text {loop }} D_{22}^{0}+\cdots \\
& =\frac{i\left(k^{2}-e^{2} \xi \hat{\phi}^{2}\right)}{k^{4}-m_{2}^{2}\left(k^{2}-\xi e^{2} \hat{\phi}^{2}\right)}
\end{aligned}
$$

with

$$
m_{2}^{2}(\hat{\phi})=\frac{\lambda}{6} \hat{\phi}^{2}+i A_{\text {loop }}=\frac{\lambda-\hat{\lambda}}{6} \hat{\phi}^{2}
$$

where $\hat{\lambda}$ is given in Eq. (2.6).

For $\phi_{1}$, this geometric summation using a vector tadpole would give rise to a mass shifting to $\frac{3 \lambda-\hat{\lambda}}{6} \hat{\phi}^{2}$. However, this is not the correct result. There are other self-energy diagrams that can contribute at order $e^{4}$. These self-energy diagrams have been computed in [4] as a momentum expansion around $p^{2}=0$. Restoring the coupling constant in logarithm, which is omitted in [4], the result, which is of the same order of $i\left(D_{11}^{0}\right)^{-1}$, can be extracted out as

$$
\Sigma_{11}=-i \frac{\hbar e^{4}}{16 \pi^{2}} \hat{\phi}^{2}\left(3+18 \ln \frac{e \hat{\phi}}{\mu}\right)
$$

Performing a summation using Eq. (3.5) we can get a dressed propagator for $\phi_{1}$ as

$$
\begin{aligned}
D_{11} & =D_{11}^{0}+D_{11}^{0} \Sigma_{11} D_{11}^{0}+D_{11}^{0} \Sigma_{11} D_{11}^{0} \Sigma_{11} D_{11}^{0}+\cdots \\
& =\frac{i}{k^{2}-m_{1}^{2}}
\end{aligned}
$$

with

$m_{1}^{2}(\hat{\phi})=\frac{\lambda}{2} \hat{\phi}^{2}+i \Sigma_{11}=\frac{\lambda}{2} \hat{\phi}^{2}+\frac{\hbar e^{4}}{16 \pi^{2}} \hat{\phi}^{2}\left(3+18 \ln \frac{e \hat{\phi}}{\mu}\right)$.

One can also check that other self-energy diagrams for $\phi_{2}$ give zero or are of higher order than the vector tadpole diagram in Fig. 2. So the correction given in Eq. (3.4) is the total leading order one-loop correction to the dressed mass of $\phi_{2}$.

Because $\left(D_{22}^{0}\right)^{-1}$, part of the whole matrix of the inverse of the propagators for $\left(\phi_{1}, \phi_{2}, A_{\mu}\right)$, has been modified to $\left(D_{22}\right)^{-1}$, other parts of propagators related to $D_{22}^{0}$ should also be modified. This can be seen clearly when solving the propagator matrix of $\left(\phi_{1}, \phi_{2}, A_{\mu}\right)$ from the matrix of the inverse propagators, as was done in [4]. The result is a shift of the $\phi_{2}$ mass to $m_{2}^{2}(\hat{\phi})$ in all these related propagators. These dressed propagators have been summarized in Appendix A.

We note that the dressed masses obtained in Eqs. (3.4) and (3.7) are consistent with the results one can obtain using the effective potential [14]. In fact, using Eqs. (2.3) one can easily verify

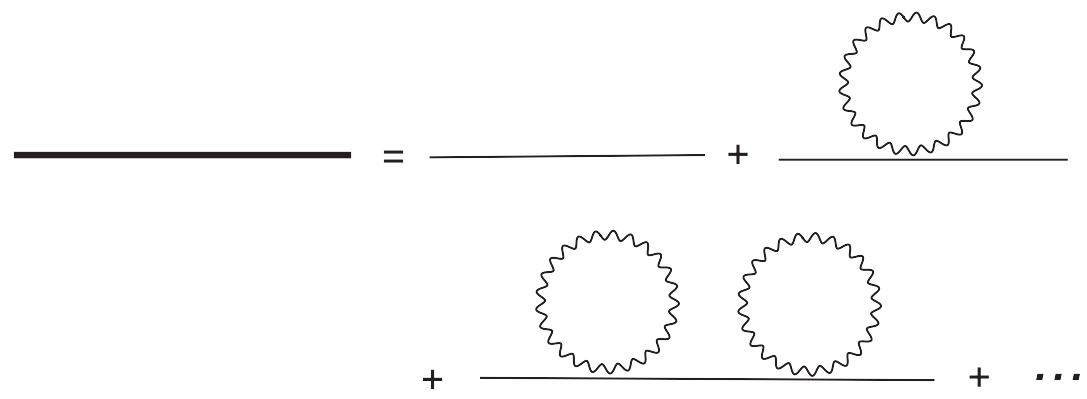

FIG. 3. The dressed propagator of $\phi_{2}$ as a resummation of a series of vector tadpole diagrams inserted into tree-level propagators. 


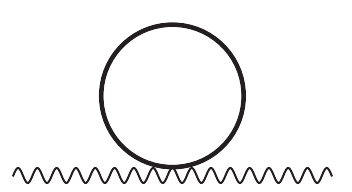

(a)

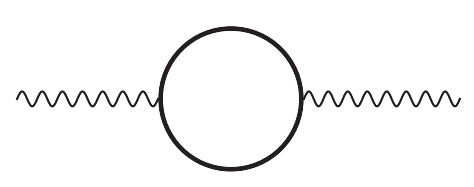

(b)

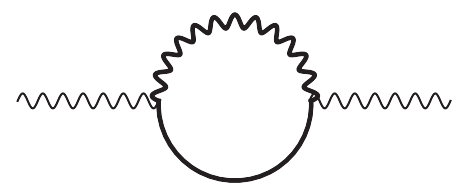

(c)

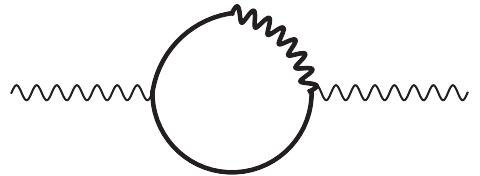

(d)

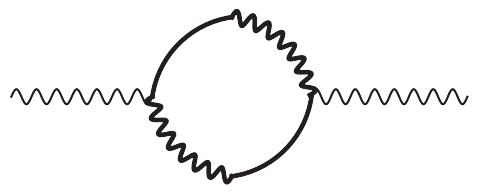

(e)

FIG. 4. One-loop self-energy diagrams for vector meson.

$$
m_{1}^{2}(\hat{\phi})=\frac{\partial^{2} V_{e^{4}}}{\partial \hat{\phi}^{2}}, \quad m_{2}^{2}(\hat{\phi})=\frac{1}{\hat{\phi}} \frac{\partial V_{e^{4}}}{\partial \hat{\phi}} .
$$

So $\phi_{2}$ becomes massless at the vacuum at the leading order determined by $V_{e^{4}}$, as it should happen as a Goldstone boson.

\section{THE VECTOR MESON MASS}

Using the dressed propagators and the vertices shown in Appendix A, we can calculate the one-loop self-energy diagrams of the vector meson and the radiative corrections to the vector meson mass. The relevant one-loop selfenergy diagrams are shown in Fig. 4 in Appendix B, and the corresponding Feynman integrals are given in Eqs. (B1), (B2), (B3), (B4), and (B5). The total self-energy $-i \Sigma_{\mu \nu}$ is the sum of the contributions of all these diagrams.

Using $\Sigma_{\mu \nu}$, we can write down the inverse of the propagator of the vector meson at one-loop order

$G_{\mu \nu}^{-1}\left(p^{2}\right)=i g_{\mu \nu}\left(p^{2}-e^{2} \hat{\phi}^{2}\right)+i \Sigma_{\mu \nu}\left(p^{2}\right)+p_{\mu} p_{\nu}$ term,

where the first term is the tree-level contribution. $\Sigma_{\mu \nu}$ can also be written as

$$
\Sigma_{\mu \nu}\left(p^{2}\right)=g_{\mu \nu} \Sigma\left(p^{2}\right)+p_{\mu} p_{\nu} \text { term. }
$$

So we can rewrite Eq. (4.1) as

$G_{\mu \nu}^{-1}\left(p^{2}\right)=i g_{\mu \nu}\left(p^{2}-e^{2} \hat{\phi}^{2}+\Sigma\left(p^{2}\right)\right)+p_{\mu} p_{\nu}$ term.

The pole mass of the vector meson, $m_{v}^{2}$, is determined by the condition that the first term in Eq. (4.3) vanishes at the point $p^{2}=m_{v}^{2}$.

$\Sigma_{\mu \nu}$ can be evaluated using a momentum expansion around the point $p^{2}=0$ as in [4]. Introducing

$$
\delta m^{2}=-\Sigma\left(p^{2}=0\right), \quad z_{2}=-\left.\frac{\partial\left(\Sigma\left(p^{2}\right)\right)}{\partial p^{2}}\right|_{p^{2}=0},
$$

and neglecting terms of higher orders in $p^{2}$, we can write Eq. (4.3) as

$G_{\mu \nu}^{-1}\left(p^{2}\right)=i g_{\mu \nu}\left[p^{2}-e^{2} \hat{\phi}^{2}-\delta m^{2}-z_{2} p^{2}\right]+p_{\mu} p_{\nu}$ term.

Detailed results to order $e^{4}$ have been given in Appendix C. Using results in Appendix $\mathrm{C}$, we can find

$\delta m^{2}=\frac{\hbar}{16 \pi^{2}} e^{4} \phi^{2}\left[\xi-\frac{5}{2}-\frac{\xi}{2} \ln \left(\frac{e^{2} \hat{\phi}^{2} \xi m_{2}^{2}}{\mu^{4}}\right)+3 \ln \frac{e^{2} \hat{\phi}^{2}}{\mu^{2}}\right]$

and

$$
z_{2}=\frac{\hbar e^{2}}{16 \pi^{2}}\left(-\frac{41}{18}+\frac{1}{3} \ln \frac{e^{2} \hat{\phi}^{2}}{\mu^{2}}\right)
$$

where terms proportional to $\Delta_{\varepsilon}$ have been subtracted in renormalization. In Eqs. (4.6) and (4.7), we have kept the coupling constants and the gauge-fixing parameter $\xi$ in the logarithms. If restoring the tree-level mass for $\phi_{1}$ and $\phi_{2}$ and neglecting the coupling constants and the gauge-fixing parameter $\xi$ in the logarithms, we agree with the results in [4].

Using Eqs. (4.5), (4.6), and (4.7), we can find the pole mass to $e^{4}$ as follows:

$$
\begin{aligned}
m_{v}^{2}= & e^{2} \hat{\phi}^{2}+\delta m^{2}+z_{2} e^{2} \hat{\phi}^{2} \\
= & e^{2} \hat{\phi}^{2}+\frac{\hbar}{16 \pi^{2}} e^{4} \hat{\phi}^{2} \\
& \times\left[\xi-\frac{43}{9}-\frac{\xi}{2} \ln \left(\frac{e^{2} \hat{\phi}^{2} \xi m_{2}^{2}}{\mu^{4}}\right)+\frac{10}{3} \ln \frac{e^{2} \hat{\phi}^{2}}{\mu^{2}}\right] .
\end{aligned}
$$

Now we set the scale $\mu=\mu_{X}$ and consider the case at vacuum $\hat{\phi}=v$. Using Eq. (2.8) and keeping terms to order $e^{4}$, we find $m_{v}^{2}(v)$ up to order $e^{4}$ 


$$
\begin{aligned}
m_{v}^{2}(v)= & e^{2} \mu_{X}^{2}+2 e^{2} \mu_{X} v_{1} \\
& +\frac{\hbar}{16 \pi^{2}} e^{4} \mu_{X}^{2}\left[\xi-\frac{43}{9}+\frac{10}{3} \ln e^{2}-\frac{\xi}{2}\left(\ln \left(\frac{e^{2} \lambda \xi}{6}\right)\right.\right. \\
& \left.\left.+\ln \left(1-\frac{\hat{\lambda}(v)}{\lambda}\right)\right)\right] .
\end{aligned}
$$

Inserting Eq. (2.16) into Eq. (4.9), it is easy to see that the $\xi$ dependence is canceled completely. We find the pole mass of the vector meson as

$m_{v}^{2}=e^{2} \mu_{X}^{2}+\frac{\hbar}{16 \pi^{2}} e^{4} \mu_{X}^{2}\left(-\frac{123}{9}+\frac{124}{9} \ln e^{2}-\frac{40}{3} \ln ^{2} e\right)$.

It is manifestly a gauge invariant result.

So far we have used a momentum expansion around $p^{2}=0$ in the evaluation of $\Sigma_{\mu \nu}\left(p^{2}\right)$ or $\Sigma\left(p^{2}\right)$. However, there is a problem in this expansion. If expanding $\Sigma\left(p^{2}\right)$ around $p^{2}=0$, such as

$$
\Sigma\left(p^{2}\right)=\Sigma(0)-z_{2} p^{2}+z_{4} p^{4}+\cdots,
$$

we hope this expansion is convergent, i.e., $\left|z_{4} p^{4}\right| \ll\left|z_{2} p^{2}\right|$ for $p^{2} \approx e^{2} \hat{\phi}^{2}$. However, the natural dimensional parameter appearing in the denominator is $e^{2} \hat{\phi}^{2}$, as we can see in Eqs. (4.6) and (4.7) that $z_{2} / \Sigma(0) \sim 1 /\left(e^{2} \hat{\phi}^{2}\right)$. We would also expect $z_{4} / z_{2} \sim 1 /\left(e^{2} \hat{\phi}^{2}\right)$. So we would find that $\left|z_{4} p^{4}\right| \sim\left|z_{2} p^{2}\right|$ for $p^{2} \approx e^{2} \hat{\phi}^{2}$ and the expansion in Eq. (4.11) may not give the correct result.

A better way to evaluate the self-energy is to do expansion around $p^{2}=e^{2} \hat{\phi}^{2}$. Introducing

$\delta \tilde{m}^{2}=-\Sigma\left(p^{2}=e^{2} \hat{\phi}^{2}\right), \quad \tilde{z}_{2}=-\left.\frac{\partial\left(\Sigma\left(p^{2}\right)\right)}{\partial p^{2}}\right|_{p^{2}=e^{2} \hat{\phi}^{2}}$,

$\Sigma\left(p^{2}\right)$ can be written as

$\Sigma\left(p^{2}\right)=-\delta \tilde{m}^{2}-\tilde{z}_{2}\left(p^{2}-e^{2} \hat{\phi}^{2}\right)+\tilde{z}_{4}\left(p^{2}-e^{2} \hat{\phi}^{2}\right)^{2}+\cdots$.

Again we would expect $\tilde{z}_{4} / \tilde{z}_{2} \sim 1 /\left(e^{2} \hat{\phi}^{2}\right)$, but the pole mass would deviate from the tree-level mass by an order $e^{4}$ correction and we would expect $p^{2}-e^{2} \hat{\phi}^{2} \sim e^{4} \hat{\phi}^{2}$ in this case. We would find that the second term in Eq. (4.13) is expected to be order $e^{6}$, and the third term in Eq. (4.13) is expected to be order $e^{8}$. Expansion to a higher order of $\left(p^{2}-e^{2} \hat{\phi}^{2}\right)^{n}$ would give corrections of order $e^{4+2 n}$. This should be a valid expansion.

Taking the first and the second terms in Eq. (4.13), we can rewrite the renormalized inverse propagator as

$$
\begin{aligned}
G_{\mu \nu}\left(p^{2}\right)^{-1}= & i g_{\mu \nu}\left[p^{2}-e^{2} \hat{\phi}^{2}-\delta \tilde{m}^{2}-\tilde{z}_{2}\left(p^{2}-e^{2} \hat{\phi}^{2}\right)\right] \\
& +p_{\mu} p_{\nu} \text { term. }
\end{aligned}
$$

The pole mass is found with the condition that the first term in Eq. (4.14) vanishes at $p^{2}=m_{v}^{2}$. This condition gives

$$
m_{v}^{2}=e^{2} \hat{\phi}^{2}+\delta \tilde{m}^{2} /\left(1-\tilde{z}_{2}\right) \approx e^{2} \hat{\phi}^{2}+\delta \tilde{m}^{2},
$$

where we have neglected correction $\tilde{z}_{2} \delta \tilde{m}^{2}$ which is of order $e^{6}$. Some details of the calculation of the self-energy diagrams in this case are given in Appendix B. We find

$\delta \tilde{m}^{2}=\frac{\hbar}{16 \pi^{2}} e^{4} \phi^{2}\left[\xi-\frac{\xi}{2} \ln \left(\frac{e^{2} \hat{\phi}^{2} \xi m_{2}^{2}}{\mu^{4}}\right)-\frac{62}{9}+\frac{10}{3} \ln \frac{e^{2} \hat{\phi}^{2}}{\mu^{2}}\right]$.

The cancellation of the gauge dependence is similar to the case in Eq. (4.10). Evaluating the mass at the vacuum and plugging Eqs. (2.8), (2.16), and (4.16) into Eq. (4.15) we get the pole mass at order $e^{4}$ as

$m_{v}^{2}=e^{2} \mu_{X}^{2}+\frac{\hbar}{16 \pi^{2}} e^{4} \mu_{X}^{2}\left(-\frac{142}{9}+\frac{124}{9} \ln e^{2}-\frac{40}{3} \ln ^{2} e\right)$.

Equation (4.17) is also a manifestly gauge invariant result, but it slightly differs from Eq. (4.10).

\section{CONCLUSION}

In summary, we have carefully calculated the one-loop self-energy contributions to the vector meson in the CW model. In our calculation, we use the dressed propagator which includes a resummation of the one-loop contribution to the scalar propagators. These contributions are found to be of the same order of the tree-level scalar propagators in the $\mathrm{CW}$ model and should be taken into account in a careful analysis. We evaluate the one-loop self-energy contribution as an expansion of momentum square both at around $p^{2}=$ 0 and at around $p^{2}=e^{2} \hat{\phi}^{2}$. The two results are slightly different, and we suggest that the latter one is the correct result because it is obtained from a more reliable expansion.

We find that our results agree with the results obtained in [4] if restoring to the tree-level scalar propagator. However, if taking the dressed propagator in calculation, the conclusion is very different from the conclusion obtained in [4]. We find that the pole mass of the vector meson calculated using the dressed propagator is gauge invariant at the vacuum determined using the effective potential calculated with a similar resummation, i.e., a resummation of daisy diagrams.

A related problem is the gauge invariance of the scalar meson mass in the CW model. This problem will be discussed in another publication. 


\section{ACKNOWLEDGMENTS}

L. W. thanks Han-qing Zheng for helpful discussion. This work is supported by National Natural Science Foundation of China (NSFC), Grant No. 11875130.

\section{APPENDIX A: DRESSED PROPAGATORS AND VERTICES}

The dressed propagators we use are

$$
\begin{gathered}
1 \longrightarrow 1=D_{11}(k)=\frac{i}{k^{2}-m_{1}^{2}}, \\
2 \frac{i\left(k^{2}-e^{2} \xi \hat{\phi}^{2}\right)}{D(k)},
\end{gathered}
$$

$$
\begin{gathered}
\mu \sim \Delta_{\mu \nu}(k)=-i\left[\frac{1}{k^{2}-e^{2} \hat{\phi}^{2}}\left(g_{\mu \nu}-\frac{k_{\mu} k_{\nu}}{k^{2}}\right)+\frac{\xi\left(k^{2}-m_{2}^{2}\right)}{D(k)} \frac{k_{\mu} k_{\nu}}{k^{2}}\right], \\
\mu \sim \sim 2=T_{\mu 2}(k)=\frac{\xi e \hat{\phi}}{D(k)} k_{\mu},
\end{gathered}
$$

where

$$
D(k)=\left(k^{2}-\beta_{1}^{2}\right)\left(k^{2}-\beta_{2}^{2}\right), \quad \beta_{1}^{2} \beta_{2}^{2}=e^{2} \hat{\phi}^{2} \xi m_{2}^{2}, \quad \beta_{1}^{2}+\beta_{2}^{2}=m_{2}^{2},
$$

and

$$
\begin{aligned}
& m_{1}^{2}(\hat{\phi})=\frac{\partial^{2} V_{e^{4}}}{\partial \hat{\phi}^{2}}=\frac{\lambda}{2} \hat{\phi}^{2}+\frac{\hbar e^{4}}{16 \pi^{2}} \hat{\phi}^{2}\left(3+18 \ln \frac{e \hat{\phi}}{\mu}\right), \\
& m_{2}^{2}(\hat{\phi})=\frac{1}{\hat{\phi}} \frac{\partial V_{e^{4}}}{\partial \hat{\phi}}=\frac{\lambda}{6} \hat{\phi}^{2}+\frac{\hbar e^{4}}{16 \pi^{2}} \hat{\phi}^{2}\left(-1+6 \ln \frac{e \hat{\phi}}{\mu}\right) .
\end{aligned}
$$

The effective vertices under a background field $\phi_{1}=\hat{\phi}$ are as follows:
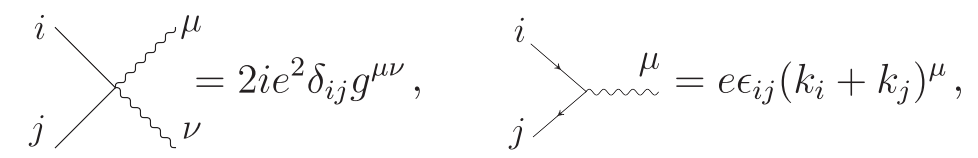

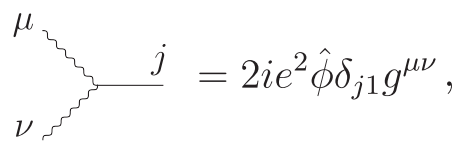

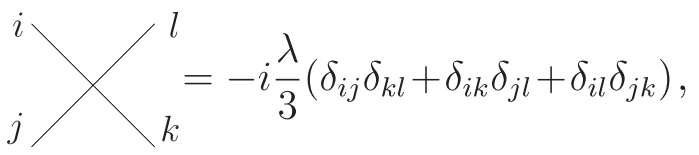

$$
Y_{k}^{i}=-i \frac{\lambda}{3} \hat{\phi}\left(\delta_{i j} \delta_{k 1}+\delta_{j k} \delta_{i 1}+\delta_{k i} \delta_{j 1}\right) .
$$




\section{APPENDIX B: EVALUATION OF ONE-LOOP SELF-ENERGY DIAGRAMS}

The corresponding Feynman integrals are

$$
\begin{gathered}
-i \Sigma_{\mu \nu}^{(a)}\left(p^{2}\right)=\frac{1}{2} \int \frac{d^{D} k}{(2 \pi)^{D}} 2 i e^{2} g_{\mu \nu}\left[D_{11}(k)+D_{22}(k)\right] \\
=\frac{1}{2} \int \frac{d^{D} k}{(2 \pi)^{D}} 2 i e^{2} g_{\mu \nu}\left[\frac{i}{k^{2}-m_{1}^{2}}+\frac{i\left(k^{2}-e^{2} \xi \hat{\phi}^{2}\right)}{D(k)}\right], \\
-i \Sigma_{\mu \nu}^{(b)}\left(p^{2}\right)=\int \frac{d^{D} k}{(2 \pi)^{D}} e \varepsilon_{12}(2 k+p)_{\mu} D_{11}(p+k) e \varepsilon_{21}(2 k+p)_{\nu} D_{22}(k) \\
=4 e^{2} \int \frac{d^{D} k}{(2 \pi)^{D}} \frac{k_{u} k_{\nu}\left(k^{2}-e^{2} \xi \hat{\phi}^{2}\right)}{\left((p+k)^{2}-m_{1}^{2}\right) D(k)}+p_{\mu} p_{\nu} \text { term, } \\
-i \Sigma_{\mu \nu}^{(c)}\left(p^{2}\right)=\int \frac{d^{D} k}{(2 \pi)^{D}} 2 i e^{2} \hat{\phi} g_{\mu \alpha} D_{11}(p+k) 2 i e^{2} \hat{\phi} g_{\beta \nu} \Delta^{\alpha \beta}(k) \\
=-4 e^{4} \hat{\phi}^{2} \int \frac{d^{D} k}{(2 \pi)^{D}}\left[\frac{g_{\mu \nu}}{\left((p+k)^{2}-m_{1}^{2}\right)\left(k^{2}-e^{2} \hat{\phi}^{2}\right)}+\frac{\xi k_{u} k_{\nu}}{\left((p+k)^{2}-m_{1}^{2}\right) D(k)}\right. \\
\left.-\frac{\xi m_{2}^{2} k_{\mu} k_{\nu}}{\left((p+k)^{2}-m_{1}^{2}\right)\left(k^{2}-e^{2} \hat{\phi}^{2}\right) k^{2}}-\frac{k_{u} k_{\nu}}{\left((p+k)^{2}-m_{1}^{2}\right) D(k) k^{2}}\right], \\
-i \Sigma_{\mu \nu}^{(d)}\left(p^{2}\right)=4 \int \frac{d^{D} k}{(2 \pi)^{D}} e \varepsilon_{21}(2 p+k)_{\mu} T_{\rho 2}(k) 2 i e^{2} \hat{\phi} g_{\nu}^{\rho} D_{11}(p+k) \\
=4 \int \frac{d^{D} k}{(2 \pi)^{D}} \frac{2 e^{4} \hat{\phi}^{2} \xi k_{\mu} k_{\nu}}{\left((p+k)^{2}-m_{1}^{2}\right) D(k)}+p_{\mu} p_{\nu} \text { term, } \\
-i \Sigma_{\mu \nu}^{(e)}\left(p^{2}\right)=0 .
\end{gathered}
$$

In these equations, the space-time dimension $D$ is $D=4-2 \varepsilon$. Notice that contributions from the second term in the parentheses in Eq. (B2), the second term in Eq. (B3), and the first term in Eq. (B4) add up to zero.

In order to simplify the calculation, we add all the above terms and get

$$
\begin{aligned}
-i \Sigma_{\mu \nu}\left(p^{2}\right)= & -i \Sigma_{\mu \nu}^{(a)}\left(p^{2}\right)+4 e^{2} \int \frac{d^{D} k}{(2 \pi)^{D}} \frac{k_{u} k_{\nu} k^{2}}{\left((p+k)^{2}-m_{1}^{2}\right) D(k)} \\
& -4 e^{4} \hat{\phi}^{2} \int \frac{d^{D} k}{(2 \pi)^{D}}\left[\frac{g_{\mu \nu}}{\left((p+k)^{2}-m_{1}^{2}\right)\left(k^{2}-e^{2} \hat{\phi}^{2}\right)}-\frac{k_{u} k_{\nu}}{\left((p+k)^{2}-m_{1}^{2}\right)\left(k^{2}-e^{2} \hat{\phi}^{2}\right) k^{2}}\right. \\
& \left.-\frac{\xi m_{2}^{2} k_{\mu} k_{\nu}}{\left((p+k)^{2}-m_{1}^{2}\right) D(k) k^{2}}\right]+p_{\mu} p_{\nu} \text { term. }
\end{aligned}
$$

After Feynman parametrization, we separate the remaining expression in Eq. (B6) into five parts and obtain

$$
-i \Sigma_{\mu \nu}\left(p^{2}\right)=-i \Sigma_{\mu \nu}^{(a)}\left(p^{2}\right)+(\mathrm{I})+(\mathrm{II})+(\mathrm{III})+(\mathrm{IV}),
$$

where $\Sigma_{\mu \nu}^{(a)}$ is independent of $p^{2}$ and is given in Eq. (C1) in Appendix C. Other terms are

$$
(\mathrm{I})=\frac{i g_{\mu \nu}}{16 \pi^{2}}\left(2 e^{4} \hat{\phi}^{2}\right) \int_{0}^{1} d x \ln \left(\frac{M^{2}\left(a_{1}, b_{1}, c_{1}\right)}{\mu^{2}}\right) M^{2}\left(-\frac{a_{1}}{c_{1}},-\frac{b_{1}}{c_{1}}, 1\right),
$$




$$
\begin{aligned}
(\mathrm{II})= & \frac{i g_{\mu \nu}}{16 \pi^{2}} e^{2} \int_{0}^{1} d x \frac{1}{d}\left[\ln \left(M^{2}(a, b, c)\right) M^{2}(a, b, c) M^{2}(5 a, 3 b, 5 c+2 d)\right. \\
& \left.-\ln \left(M^{2}(a, b-d, c+d)\right) M^{2}(a, b-d, c+d) M^{2}(5 a, 3 b-3 d, 5 c+3 d)\right], \\
(\mathrm{III})= & \frac{i g_{\mu \nu}}{16 \pi^{2}} e^{2}\left[\int_{0}^{1} d x_{1} \int_{0}^{1-x_{1}} d x_{2} 4 \hat{M}^{2}+\int_{0}^{1} d x\left(2 e^{2} \hat{\phi}^{2}\right)(1-x)\right. \\
& \left.-\int_{0}^{1} d x\left(\frac{3}{2 d}\left(\left(M^{2}(a, b, c)\right)^{2}-\left(M^{2}(a, b-d, c+d)\right)^{2}\right)-2 a x^{2}(1-x)\right)\right], \\
(\mathrm{IV})= & \frac{i g_{\mu \nu}}{16 \pi^{2}} e^{2} \Delta_{\varepsilon}\left[-4 e^{2} \hat{\phi}^{2}+\int_{0}^{1} d x_{1} \int_{0}^{1-x_{1}} d x_{2}\left(6 \hat{M}^{2}+2 l^{2}+2 e^{2} \hat{\phi}^{2}\right)\right],
\end{aligned}
$$

where

$$
\begin{aligned}
M^{2}(a, b, c) & =a x^{2}+b x+c, \quad \hat{M}^{2}=a x_{1}^{2}+b x_{1}+c+d x_{2}, \quad l^{2}=a x_{1}^{2}, \\
a & =p^{2}, \quad b=m_{1}^{2}-\beta_{2}^{2}-p^{2}, \quad c=\beta_{2}^{2}, \quad d=\beta_{1}^{2}-\beta_{2}^{2}, \\
a_{1} & =p^{2}, \quad b_{1}=m_{1}^{2}-e^{2} \hat{\phi}^{2}-p^{2}, \quad c_{1}=e^{2} \hat{\phi}^{2},
\end{aligned}
$$

and

$$
\Delta_{\varepsilon}=\frac{1}{\varepsilon}-\gamma_{E}+\ln 4 \pi
$$

In Eqs. (B7), (B8), (B9), (B10), and (B11), we have neglected all terms proportional to $p_{\mu} p_{\nu}$. So far we have not made an approximation in the evaluation of self-energy diagrams except neglecting terms proportional to $p_{\mu} p_{\nu}$. A further examination shows that the last term in Eq. (B3) gives a higher-order contribution that can be neglected in our analysis. If neglecting this contribution, Eqs. (B8) and (B9) would be rewritten as other forms.

Notice that two terms in the bracket in Eq. (B9) cancel with each other when $d=0$. When $d$ is small compared to other parameters, these two terms would give a result proportional to a factor $d$ which cancels the $d$ in the denominator outside the bracket. This is the case for $p^{2} \approx e^{2} \hat{\phi}^{2}$. A careful calculation should be done to extract the result in this case. After a careful calculation, an expression for the one-loop contribution to the self-energy of the vector meson is found at $p^{2} \approx e^{2} \hat{\phi}^{2}$ as

$$
\begin{aligned}
-i \Sigma_{\mu \nu}\left(p^{2}\right)= & \frac{i \hbar g_{\mu \nu}}{16 \pi^{2}}\left[e^{4} \phi^{2}\left(\xi-\frac{\xi}{2} \ln \left(\frac{e^{2} \hat{\phi}^{2} \xi m_{2}^{2}}{\mu^{4}}\right)-\frac{62}{9}+\frac{10}{3} \ln \frac{e^{2} \hat{\phi}^{2}}{\mu^{2}}\right)\right. \\
& \left.+\left(\frac{31}{9}-\frac{5}{3} \ln \frac{e \hat{\phi}^{2}}{\mu^{2}}+2 \ln \frac{m_{1}^{2}}{\mu^{2}}\right) e^{2}\left(p^{2}-e^{2} \hat{\phi}^{2}\right)\right],
\end{aligned}
$$

where we have eliminated terms proportional to $\Delta_{\varepsilon}$ by subtraction and neglected terms proportional to $p_{\mu} p_{\nu}$. We have also restored a factor $\hbar$ in Eq. (B14) for a one-loop correction. Using Eq. (B14), we can find

$$
\delta \tilde{m}^{2}=\frac{\hbar}{16 \pi^{2}} e^{4} \phi^{2}\left[\xi-\frac{\xi}{2} \ln \left(\frac{e^{2} \hat{\phi}^{2} \xi m_{2}^{2}}{\mu^{4}}\right)-\frac{62}{9}+\frac{10}{3} \ln \frac{e^{2} \hat{\phi}^{2}}{\mu^{2}}\right]
$$

and

$$
\tilde{z}_{2}=\frac{\hbar e^{2}}{16 \pi^{2}}\left(\frac{31}{9}-\frac{5}{3} \ln \frac{e \hat{\phi}^{2}}{\mu^{2}}+2 \ln \frac{m_{1}^{2}}{\mu^{2}}\right) .
$$

We note that when evaluating the self-energy diagrams in Fig. 4 at $p^{2}=e^{2} \hat{\phi}^{2}$, we would find imaginary parts in some of the diagrams. There is an imaginary part in Fig. 4(b) that does not depend on the gauge-fixing parameter $\xi$. At the order we are considering, this imaginary part is canceled by a contribution from Fig. 4(c). Other contributions to the imaginary parts are proportional to the gauge-fixing parameter $\xi$. These terms add up to zero and the imaginary parts cancel completely. So we do not have an imaginary part in the final result of the self-energy of the vector meson. 


\section{APPENDIX C: SUMMARY OF ONE-LOOP SELF-ENERGY CONTRIBUTIONS}

The self-energy contributions given in Eqs. (B1), (B2), (B3), (B4), and (B5) can be evaluated as an expansion of $p^{2}$ around $p^{2}=0$. The results to order $p^{2}$ are

$$
\begin{aligned}
& -i \Sigma_{\mu \nu}^{(a)}\left(p^{2}\right)=\frac{i g_{\mu \nu}}{16 \pi^{2}}\left[e^{4} \xi \phi^{2}+e^{4} \xi \phi^{2}\left(\Delta_{\varepsilon}-\frac{1}{2} \ln \left(\frac{e^{2} \hat{\phi}^{2} \xi m_{2}^{2}}{\mu^{4}}\right)\right)\right], \\
& -i \Sigma_{\mu \nu}^{(b)}\left(p^{2}\right)=\frac{i g_{\mu \nu}}{16 \pi^{2}}\left[-\frac{3}{2} e^{4} \hat{\phi}^{2} \xi+\left(-\frac{5}{18}+\frac{2 m_{1}^{2}}{3 m_{2}^{2}}\right) e^{2} p^{2}\right. \\
& \left.-\left(e^{4} \hat{\phi}^{2} \xi+\frac{1}{3} e^{2} p^{2}\right)\left(\Delta_{\varepsilon}-\frac{1}{2} \ln \left(\frac{e^{2} \hat{\phi}^{2} \xi m_{2}^{2}}{\mu^{4}}\right)\right)\right] \text {, } \\
& -i \Sigma_{\mu \nu}^{(c)}\left(p^{2}\right)=\frac{i g_{\mu \nu}}{16 \pi^{2}}\left[-\left(\frac{5}{2}+\frac{3}{2} \xi\right) e^{4} \hat{\phi}^{2}-2\left(1-\frac{m_{1}^{2}}{3 m_{2}^{2}}-\frac{1}{6} \ln \frac{e^{2} \hat{\phi}^{2}}{\mu^{2}}+\frac{1}{12} \ln \left(\frac{e^{2} \hat{\phi}^{2} \xi m_{2}^{2}}{\mu^{4}}\right)\right) e^{2} p^{2}\right. \\
& \left.-(3+\xi) e^{4} \hat{\phi}^{2} \Delta_{\varepsilon}+e^{4} \hat{\phi}^{2}\left(3 \ln \frac{e^{2} \hat{\phi}^{2}}{\mu^{2}}+\frac{1}{2} \xi \ln \left(\frac{e^{2} \hat{\phi}^{2} \xi m_{2}^{2}}{\mu^{4}}\right)\right)\right] \text {, } \\
& -i \Sigma_{\mu \nu}^{(d)}\left(p^{2}\right)=\frac{i g_{\mu \nu}}{16 \pi^{2}}\left[3 e^{4} \hat{\phi}^{2} \xi-\frac{4 m_{1}^{2}}{3 m_{2}^{2}} e^{2} p^{2}+2 e^{4} \hat{\phi}^{2} \xi\left(\Delta_{\varepsilon}-\frac{1}{2} \ln \left(\frac{e^{2} \hat{\phi}^{2} \xi m_{2}^{2}}{\mu^{4}}\right)\right)\right], \\
& -i \Sigma_{\mu \nu}^{(e)}\left(p^{2}\right)=0
\end{aligned}
$$

In these results, we have neglected all terms proportional to $p_{\mu} p_{\nu}$. We note that $-i \Sigma_{\mu \nu}^{(a)}$ is independent of $p^{2}$, so we have $-i \Sigma_{\mu \nu}^{(a)}(0)=-i \Sigma_{\mu \nu}^{(a)}\left(e^{2} \hat{\phi}^{2}\right)$.

Summarizing results in Eqs. (C1), (C2), (C3), (C4), and (C11), we find the one-loop contribution to the self-energy as an expansion at $p^{2}=0$ as

$$
\begin{aligned}
-i \Sigma_{\mu \nu}\left(p^{2}\right)= & \frac{i \hbar g_{\mu \nu}}{16 \pi^{2}}\left[e^{4} \phi^{2}\left(\xi-\frac{5}{2}-\frac{\xi}{2} \ln \left(\frac{e^{2} \hat{\phi}^{2} \xi m_{2}^{2}}{\mu^{4}}\right)+3 \ln \frac{e^{2} \hat{\phi}^{2}}{\mu^{2}}\right)\right. \\
& \left.+\left(-\frac{41}{18}+\frac{1}{3} \ln \frac{e^{2} \hat{\phi}^{2}}{\mu^{2}}\right) e^{2} p^{2}\right]
\end{aligned}
$$

where we have eliminated terms proportional to $\Delta_{\varepsilon}$ by subtraction and neglected terms proportional to $p_{\mu} p_{\nu}$. We have also restored a factor $\hbar$ in Eq. (C6) for a one-loop correction.

If we do not use the dressed propagator of scalars and restore to the tree-level propagator, we just need to take $m_{1}^{2}=\frac{\lambda}{2} \hat{\phi}^{2}$ and $m_{2}^{2}=\frac{\lambda}{6} \hat{\phi}^{2}$. If taking the tree-level masses of the scalar mesons and neglecting the coupling constants and the gaugefixing parameter $\xi$ in logarithms, our results agree with the results in [4].

For $p^{2} \approx e^{2} \phi^{2}$, we can find the self-energy contributions as follows:

$$
\begin{aligned}
& -i \Sigma_{\mu \nu}^{(a)}\left(p^{2}\right)=\frac{i g_{\mu \nu}}{16 \pi^{2}}\left[e^{4} \xi \phi^{2}+e^{4} \xi \phi^{2}\left(\Delta_{\varepsilon}-\frac{1}{2} \ln \left(\frac{e^{2} \hat{\phi}^{2} \xi m_{2}^{2}}{\mu^{4}}\right)\right)\right], \\
& -i \Sigma_{\mu \nu}^{(b)}\left(p^{2}\right)=\frac{i g_{\mu \nu}}{16 \pi^{2}}\left[-\frac{e^{2} p^{2}}{3} \Delta_{\varepsilon}-\frac{8}{9} e^{2} p^{2}+\frac{1}{3} e^{2} p^{2} \ln \frac{p^{2}}{\mu^{2}}+\frac{1}{3} i \pi e^{2} p^{2}\right. \\
& \left.-e^{4} \xi \hat{\phi}^{2}\left(\Delta_{\varepsilon}-2-\ln \frac{p^{2}}{\mu^{2}}-i \pi\right)\right] \text {, } \\
& -i \Sigma_{\mu \nu}^{(c)}\left(p^{2}\right)=(\mathrm{I})+\frac{i g_{\mu \nu}}{16 \pi^{2}}\left[e^{4} \hat{\phi}^{2}\left(1-3 \Delta_{\varepsilon}\right)+\frac{5}{9} e^{2} p^{2}-\frac{1}{3} e^{2} p^{2} \ln \frac{p^{2}}{\mu^{2}}-\frac{1}{3} i \pi e^{2} p^{2}\right. \\
& \left.-e^{4} \xi \hat{\phi}^{2}\left(\Delta_{\varepsilon}-2-\ln \frac{p^{2}}{\mu^{2}}-i \pi\right)\right] \text {, }
\end{aligned}
$$




$$
\begin{gathered}
-i \Sigma_{\mu \nu}^{(d)}\left(p^{2}\right)=\frac{i g_{\mu \nu}}{16 \pi^{2}}\left[e^{4} \xi \hat{\phi}^{2}\left(2 \Delta_{\varepsilon}-4-2 \ln \frac{p^{2}}{\mu^{2}}-2 i \pi\right)\right], \\
-i \Sigma_{\mu \nu}^{(e)}\left(p^{2}\right)=0
\end{gathered}
$$

where

$$
\begin{aligned}
(\mathrm{I})= & \frac{i g_{\mu \nu}}{16 \pi^{2}}\left(e^{4} \hat{\phi}^{2}\right)\left[\left(-\frac{20}{3}-\frac{5 p^{2}}{9 e^{2} \hat{\phi}^{2}}-\frac{e^{2} \hat{\phi}^{2}}{3 p^{2}}\right)+\left(\frac{3}{2}+\frac{p^{2}}{6 e^{2} \hat{\phi}^{2}}+\frac{3 e^{2} \phi^{2}}{2 p^{2}}+\frac{e^{4} \hat{\phi}^{4}}{6 p^{4}}\right) \ln \left(\frac{e^{2} \hat{\phi}^{2}}{\mu^{2}}\right)\right. \\
& +\left(\frac{3}{2}+\frac{p^{2}}{6 e^{2} \hat{\phi}^{2}}-\frac{3 e^{2} \phi^{2}}{2 p^{2}}-\frac{e^{4} \hat{\phi}^{4}}{6 p^{4}}\right) \ln \left(\frac{m_{1}^{2}}{\mu^{2}}\right) \\
& \left.+\left(-\frac{5}{3}-\frac{p^{2}}{6 e^{2} \hat{\phi}^{2}}-\frac{e^{2} \hat{\phi}^{2}}{6 p^{2}}\right)\left(x_{+}-x_{-}\right) \ln \left(\frac{x_{+}+x_{+} x_{-}}{x_{-}+x_{+} x_{-}}\right)\right]
\end{aligned}
$$

with

$$
x_{+}+x_{-}=\frac{m_{1}^{2}-e^{2} \hat{\phi}^{2}-p^{2}}{p^{2}}, \quad x_{-} x_{+}=\frac{e^{2} \phi^{2}}{p^{2}} .
$$

Again, we have neglected all terms proportional to $p_{\mu} p_{\nu}$ in Eqs. (C8), (C9), and (C10). Using Eqs. (C7), (C8), (C9), and (C10), we can obtain $\Sigma_{\mu \nu}$ as an expansion around $p^{2}=e^{2} \phi^{2}$ and get Eq. (B14).

[1] S. R. Coleman and E. J. Weinberg, Radiative corrections as the origin of spontaneous symmetry breaking, Phys. Rev. D 7, 1888 (1973).

[2] R. Jackiw, Functional evaluation of the effective potential, Phys. Rev. D 9, 1686 (1974).

[3] N. K. Nielsen, On the gauge dependence of spontaneous symmetry breaking in gauge theories, Nucl. Phys. B101, 173 (1975).

[4] J. S. Kang, Gauge invariance of the scalar-vector mass ratio in the Coleman-Weinberg model, Phys. Rev. D 10, 3455 (1974).

[5] N. K. Nielsen, Gauge dependence of effective potential and self consistent dimensional reduction, Z. Phys. C 33, 579 (1987).

[6] D. Bazeia, Gauge invariance and daisies, Phys. Lett. B 207, 53 (1988).

[7] D. Johnston, Coleman-Weinberg, Nielsen, and Daisies, Phys. Lett. B 186, 185 (1987).

[8] A. L. Larsen and N.K. Nielsen, Vector tadpole graphs in the Coleman-Weinberg model, Z. Phys. C 44, 689 (1989).

[9] A. Andreassen, W. Frost, and M. D. Schwartz, Consistent use of effective potentials, Phys. Rev. D 91, 016009 (2015).

[10] J. R. Espinosa, M. Garny, and T. Konstandin, Interplay of infrared divergences and gauge-dependence of the effective potential, Phys. Rev. D 94, 055026 (2016).
[11] M. Quiros, On daisy and superdaisy resummation of the effective potential at finite temperature, in 4th Hellenic School on Elementary Particle Physics, 1992 [arXiv:hep$\mathrm{ph} / 9304284]$.

[12] C. Wainwright, S. Profumo, and M. J. Ramsey-Musolf, Gravity waves from a cosmological phase transition: Gauge artifacts and daisy resummations, Phys. Rev. D 84, 023521 (2011).

[13] M. Garny and T. Konstandin, On the gauge dependence of vacuum transitions at finite temperature, J. High Energy Phys. 07 (2012) 189.

[14] D. Metaxas and E. J. Weinberg, Gauge independence of the bubble nucleation rate in theories with radiative symmetry breaking, Phys. Rev. D 53, 836 (1996).

[15] A. D. Plascencia and C. Tamarit, Convexity, gauge-dependence and tunneling rates, J. High Energy Phys. 10 (2016) 099.

[16] L. Di Luzio and L. Mihaila, On the gauge dependence of the Standard Model vacuum instability scale, J. High Energy Phys. 06 (2014) 079.

[17] A. Andreassen, W. Frost, and M. D. Schwartz, Consistent Use of the Standard Model Effective Potential, Phys. Rev. Lett. 113, 241801 (2014).

[18] S. Chigusa, T. Moroi, and Y. Shoji, Decay rate of electroweak vacuum in the Standard Model and beyond, Phys. Rev. D 97, 116012 (2018). 
[19] S. Chigusa, T. Moroi, and Y. Shoji, State-of-the-Art Calculation of the Decay Rate of Electroweak Vacuum in the Standard Model, Phys. Rev. Lett. 119, 211801 (2017).

[20] S. H. H. Tye and Y. Vtorov-Karevsky, Effective action of spontaneously broken gauge theories, Int. J. Mod. Phys. A 13, 95 (1998).

[21] A. Akil and X. Tong, Gauge invariance, polar coordinates and inflation, J. High Energy Phys. 03 (2021) 096.
[22] D. Metaxas, Gauge independence of the physical fermion mass in the effective action of theories with radiative symmetry breaking, arXiv:2010.07044.

[23] S. P. Martin, Taming the Goldstone contributions to the effective potential, Phys. Rev. D 90, 016013 (2014).

[24] J. Elias-Miro, J. R. Espinosa, and T. Konstandin, Taming infrared divergences in the effective potential, J. High Energy Phys. 08 (2014) 034. 